\title{
CECOSTOMÍA AMPLIA: UNA ALTERNATIVA DE PROTECCIÓN DE UNA ANASTOMOSIS COLORRECTAL BAJA EN CÁNCER DE RECTO*
}

\author{
Drs. Guillermo Bannura C. ${ }^{1}$, Alejandro Barrera E. ${ }^{1}$, Carlos Melo L. ${ }^{1}$, \\ Felipe Illanes F. ${ }^{1}$, Cristian Gallardo V. ${ }^{1}$, Cristóbal Suazo L. ${ }^{1}$ \\ 1 Servicio y Departamento de Cirugía, Hospital Clínico San Borja Arriarán, Campus Centro, \\ Facultad de Medicina, Universidad de Chile. \\ Chile.
}

\begin{abstract}
Enlarged cecostomy as an alternative for the protection of low colorrectal anastomoses

Background: Loop ileostomy, usually used as protection for low colorrectal anastomoses, has a significant number of complications. Aim: To assess the results of a new technique, called enlarged cecostomy for anastomotic protection. Material and Methods: The enlarged cecostomy technique, described herein, was applied to 12 consecutive patients, subjected to a radical resection for a low rectal cancer. Most procedures were performed after a neo-adjuvant therapy. The results obtained in these patients were compared with similar series of patients in whom a loop ileostomy was performed. Results: 4 low anterior resections and 8 ultra-low anterior resections were performed among patients subjected to enlarged cecostomy (11 laparoscopic). Among patients subjected to loop ileostomy, 8 low anterior resections and 4 ultra-low anterior resections were performed (4 laparoscopic). The execution lapse for enlarged cecostomy was 15 minutes and for loop ileostomy, 25 minutes. Patients subjected to cecostomy had a lower hospitalization time. This was because 4 patients had a dysfunction of the loop ileostomy. The degree of diversion was complete in 11 patients subjected to enlarged cecostomy. The ostomy was closed in 7 patients subjected to loop ileostomy and the same number of patients subjected to enlarged cecostomy. Conclusions: Enlarged cecostomy is a valid protection alternative for patients operated for low rectal cancer. It is easier to perform than loop ileostomy.
\end{abstract}

Key words: Rectal cancer, cecostomy, ileostomy.

\section{Resumen}

Antecedentes: La ileostomía en asa (IA) se utiliza en forma habitual como protección de una anastomosis colorrectal baja, técnica que se asocia a una morbilidad no despreciable. El objetivo de este estudio es

\footnotetext{
Los autores no refieren conflictos de interés.

Correspondencia: Guillermo Bannura C.

gbannura@gtdmail.com
}

*Recibido el 12 de diciembre de 2013 y aceptado para publicación el 9 de enero de 2014. 
evaluar los resultados de una técnica alternativa con los mismos fines de protección denominada cecostomía amplia (CA). Pacientes y Método: Se describe en detalle la técnica de la CA, que se aplica en 12 pacientes consecutivos sometidos a una resección radical por cáncer de recto bajo, la mayoría luego de neoadyuvancia. Se compara esta serie con una serie similar de pacientes en los cuales se utilizó como protección una IA. Resultados: Se realizaron 4 RAB y 8 RAUB en el grupo CA (11 por vía laparoscópica) y 8 RAB y 4 RAUB (4 por laparoscopía). El tiempo de ejecución promedio de la CA fue 15 min versus 25 min de la IA. La única diferencia estadísticamente significativa fue el tiempo de hospitalización a favor de la cecostomía $(\mathrm{p}<0,001)$, lo que se explica por la disfunción ileostómica en 4 pacientes sometidos a una IA. El grado de desfuncionalización fue completo en 11 pacientes con una CA. Se efectuó el cierre de la ostomía en 14 pacientes ( 7 en cada grupo) con un tiempo de hospitalización promedio de 3,5 días (extremos 2-5) para el grupo CA y 5,3 días (extremos 4-8) para el grupo IA $(\mathrm{p}<0,05)$. Conclusión: la CA se aprecia como una alternativa válida como método de protección de una anastomosis colorrectal en pacientes intervenidos por un cáncer de recto bajo, con algunas ventajas sobre la IA por la facilidad en la ejecución y en el cierre y probablemente una reducción de la morbilidad específica. Se requiere un estudio aleatorio con un número mayor de pacientes para confirmar estos resultados.

Palabras clave: Ostomía de protección, cecostomía, cáncer de recto bajo.

\section{Introducción}

La protección selectiva de una anastomosis colorrectal baja es una estrategia habitual en el manejo del cáncer de recto bajo, especialmente luego de radioterapia preoperatoria y en pacientes varones, con el fin de mitigar las consecuencias sépticas de una dehiscencia anastomótica ${ }^{1}$. Aunque no existe evidencia científica contundente, la ileostomía en asa (IA) desplazó a la colostomía transversa derecha en asa, probablemente por ser mejor tolerada por los pacientes y tener un menor riesgo relativo de prolapso $^{2}$. Sin embargo, la IA no está exenta de morbilidad, tanto en la confección como en el cierre. En una revisión sistemática sobre 6.107 casos de cierre de ileostomía en asa efectuado en Inglaterra, la morbilidad global alcanzó el $17,3 \%$ y la mortalidad $0,4 \%{ }^{3}$, cifra similar a lo publicado en Chile ${ }^{4,5}$.

El objetivo de este estudio es dar a conocer una técnica alternativa de protección de una anastomosis colorrectal baja en paciente intervenidos por un cáncer de recto bajo que hemos denominado cecostomía amplia (CA). Se compara los resultados clínicos de la técnica con una serie similar de pacientes sometidos a una ileostomía en asa (IA) con los mismos fines de protección.

\section{Técnica quirúrgica}

Una vez completado el tiempo de la confección de una anastomosis colorrectal baja, ésta se controla mediante rectoscopio rígido para medir la altura y evaluar la hemostasia y la hermeticidad. Si se establece la necesidad de protección de la anastomosis, se realiza una incisión de contrabertura en la fosa ilíaca derecha o en el flanco derecho de 4 a $6 \mathrm{~cm}$ de longitud, de acuerdo a la contextura del paciente, en la unión del recto anterior con los músculos anchos. Luego se procede a la apertura de la aponeurosis en cruz con divulsión de los músculos y apertura del peritoneo que permita pasar con facilidad dos dedos. Exteriorización del ciego, previa apendicectomía, cierre de la laparotomía o de la mini-incisión laparoscópica, colotomía de 3 a $4 \mathrm{~cm}$ y "maduración" de la cecostomía con una corona de puntos de Vycril 3/0 de pared total en el ciego que se fijan a la piel. No se emplea ningún medio de fijación externo como bagueta o puente cutáneo. Aplicación de la prótesis de ostomía habitual. El aspecto externo es indistinguible de cualquier colostomía (Figura 1). Excepcionalmente puede requerir una liberación del ciego para que se exteriorice con mayor holgura. La técnica de la CA se puede realizar con igual facilidad en cirugía abierta o laparoscópica.

\section{Pacientes y Método}

En un período de 14 meses se utilizó la técnica de CA en forma consecutiva en 12 pacientes sometidos a una resección anterior baja (RAB) o ultrabaja (RAUB) por un cáncer de recto bajo, cuyas características demográficas se comparan con 12 pacientes similares protegidos mediante una IA en el período de 15 meses (Tabla 1). Previo a la radioterapia, el tumor era palpable en 12 pacientes del grupo CA y en 11 del grupo IA. Once pacientes del grupo CA y 9 del grupo IA fueron sometidos al esquema largo de neoadyuvancia con radioquimioterapia y sometidos a cirugía radical a los 3 meses, según protocolo ${ }^{6}$.

Una anastomosis a $8 \mathrm{~cm}$ o menos del margen anal se define como RAB y por debajo de $5 \mathrm{~cm}$ como RAUB. La dehiscencia anastomótica se definió de acuerdo a un estudio previo ${ }^{1}$. Se define como morbilidad mayor aquella que prolonga la estadía hospitalaria o requiere una re-intervención (Dindo- 


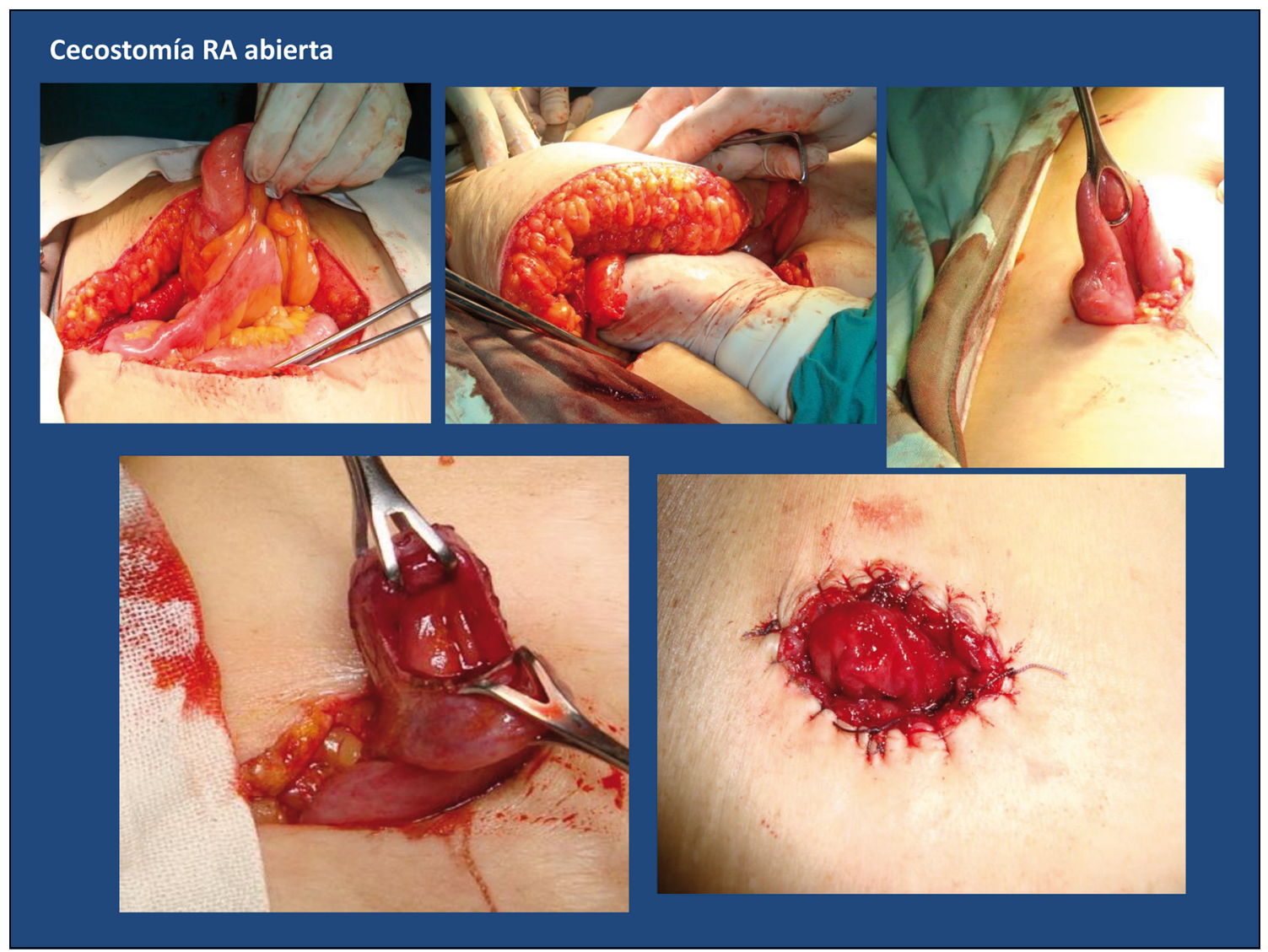

Figura 1. Exteriorización, apertura y maduración del ciego (abierta).

Clavien Grade III) ${ }^{7}$. La disfunción ileostómica es una forma de íleo postoperatorio que se caracteriza por la ausencia de gases y contenido líquido por

Tabla 1. Características demográficas según tipo de ostomía de protección

\begin{tabular}{|lcc|}
\hline Variable & $\begin{array}{c}\text { Cecostomía } \\
\mathbf{n = 1 2}\end{array}$ & $\begin{array}{c}\text { Ileostomía } \\
\mathbf{n}=\mathbf{1 2}\end{array}$ \\
$\begin{array}{l}\text { Edad promedio } \\
\text { (extremos) años }\end{array}$ & $60,6(36-83)$ & $59,9(36-82)$ \\
$\begin{array}{l}\text { Género (M/H) } \\
\text { Albúmina promedio } \\
\text { (extremos) gr/dl }\end{array}$ & $4,25(3,4-4,9)$ & $3,98(3,4-4,9)$ \\
$\begin{array}{l}\text { Hematocrito promedio } \\
\text { (extremos) \% }\end{array}$ & $39,4(34-43)$ & $37,9(34-43)$ \\
$\begin{array}{l}\text { CEA preoperatorio } \\
>10 \text { ng/ml n }\end{array}$ & 3 & 5 \\
$\begin{array}{l}\text { Enfermedades } \\
\text { asociadas }\end{array}$ & 5 & 5 \\
\hline
\end{tabular}

la ileostomía durante 3 a 5 días, acompañado de distensión abdominal y mala tolerancia a la realimentación oral. Luego se produce una descarga de grandes volúmenes de contenido intestinal de color verdoso oscuro ("verde botella") de 1,2 a 2 litros por día durante 3 a 7 días, a pesar de lo cual el paciente persiste distendido y con mala tolerancia oral, período en el cual requiere una vigorosa reposición de volumen y electrolíticos. Finalmente, el cuadro progresa espontáneamente a la mejoría, con disminución de los débitos de la ostomía, cambio de la coloración y de la consistencia de las deposiciones (heces cremosas de color amarillento y luego de color café claro), con la aparición de gases en la bolsa, el aplanamiento del abdomen y la progresiva tolerancia a la realimentación oral.

Para evaluar el grado de desfuncionalización logrado por la CA se hizo un cuestionario a los pacientes consultando sobre la frecuencia y el tipo de material eliminado por el ano en el postoperatorio hasta el cierre de la CA o al corte del estudio. 


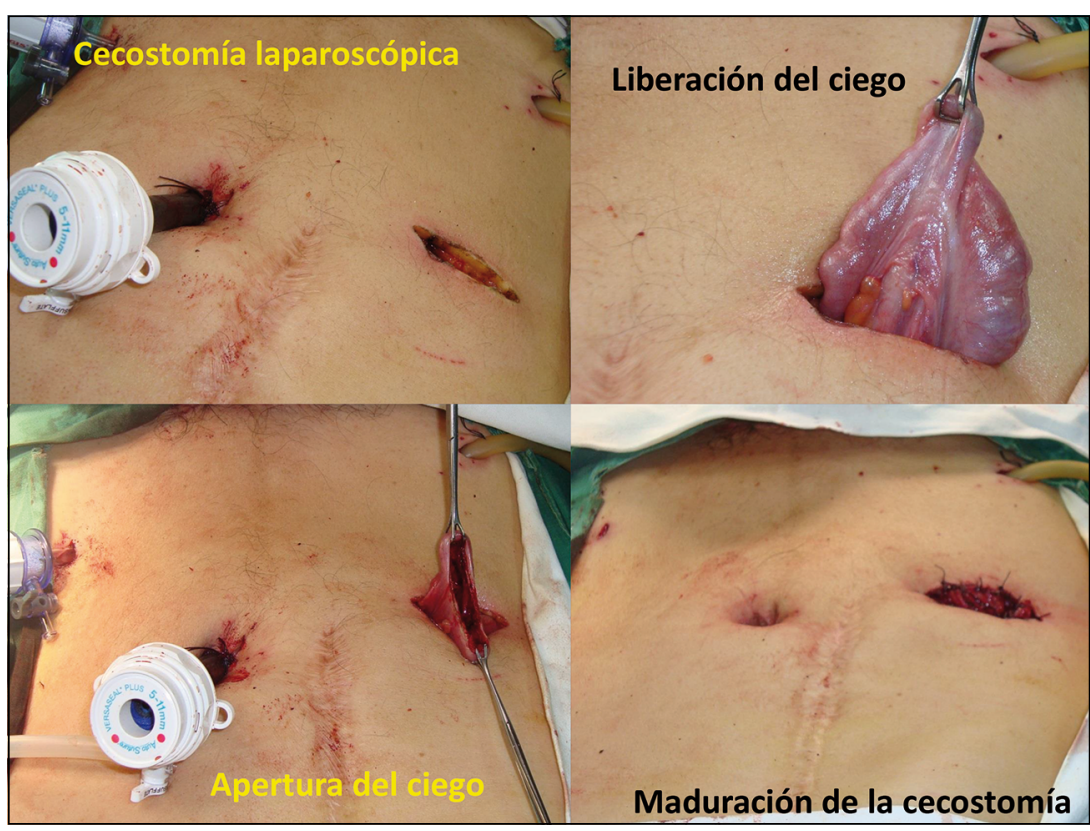

Figura 2. Exteriorización, apertura y maduración del ciego (laparoscópica).

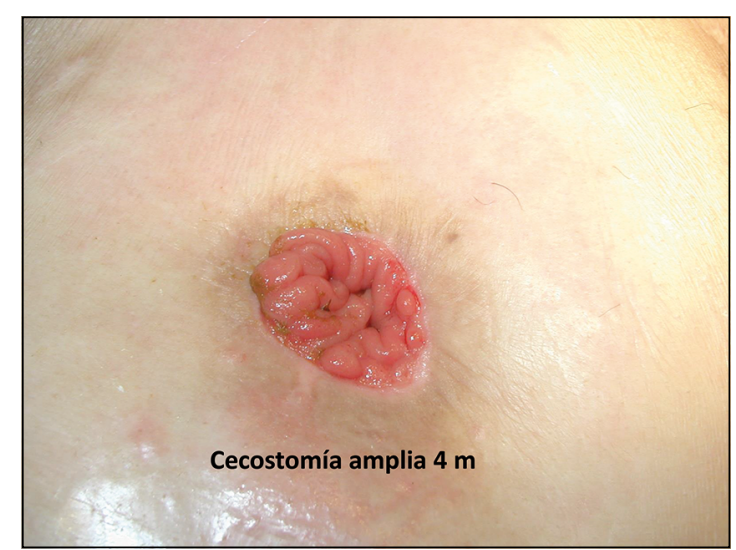

Figura 3. Aspecto de la cecostomía amplia a los 4 meses.

\section{Resultados}

Se realizaron 4 RAB y 8 RAUB en el grupo CA, 1 por vía abierta (con exenteración posterior) y 11 por vía laparoscópica (2 convertidas). En el grupo IA se efectuaron 8 RAB y 4 RAUB, 8 por laparotomía y 4 con abordaje mínimamente invasivo ( $\sin$ conversiones). El tiempo de ejecución promedio de la CA fue 15 min versus 25 min de la IA.

No hubo diferencias estadísticamente significativas entre ambos grupos de pacientes en cuanto a edad, género, compromiso del estado general o estadio oncológico. Los resultados quirúrgicos inmediatos y la etapificación definitiva se aprecian en la Tabla 2. Hubo 2 casos de respuesta patológica completa en cada grupo. La única diferencia estadísticamente significativa fue el tiempo de hospitalización a favor de la cecostomía $(\mathrm{p}<0,001)$, lo que se explica por la disfunción ileostómica con íleo postoperatorio severo que ocurrió en 4 pacientes sometidos a una IA de protección. La dehiscencia anastomótica ocurrió en 3 pacientes en cada grupo, con débitos escasos y con repercusión sistémica en un paciente de cada grupo.

El intervalo entre la operación principal y el cierre de la cecostomía fue de 6 meses como promedio (extremos 3-7). En la evaluación del grado de desfuncionalización de la CA, 1 paciente refiere haber tenido evacuaciones diarias por el ano, 2 pacientes registraron la eliminación de una excreción mucosa intermitente escasa por el ano y 9 señalan ausencia de evacuaciones, de excreciones o de gases por el ano.

\section{Cierre de la ostomía}

En el período de estudio, 14 pacientes (7 en cada grupo) se han reconstituido el tránsito intestinal. En 2 casos con CA para el cierre se utilizó un lineal cortante $80 \mathrm{~mm}$, reforzada por una sutura corrida de Vycril 3/0. En los 5 pacientes restantes con una CA el cierre fue manual con el mismo material en 2 planos. En los 7 casos de IA se practicó una anastomosis grapada terminal funcional. El tiempo operatorio 


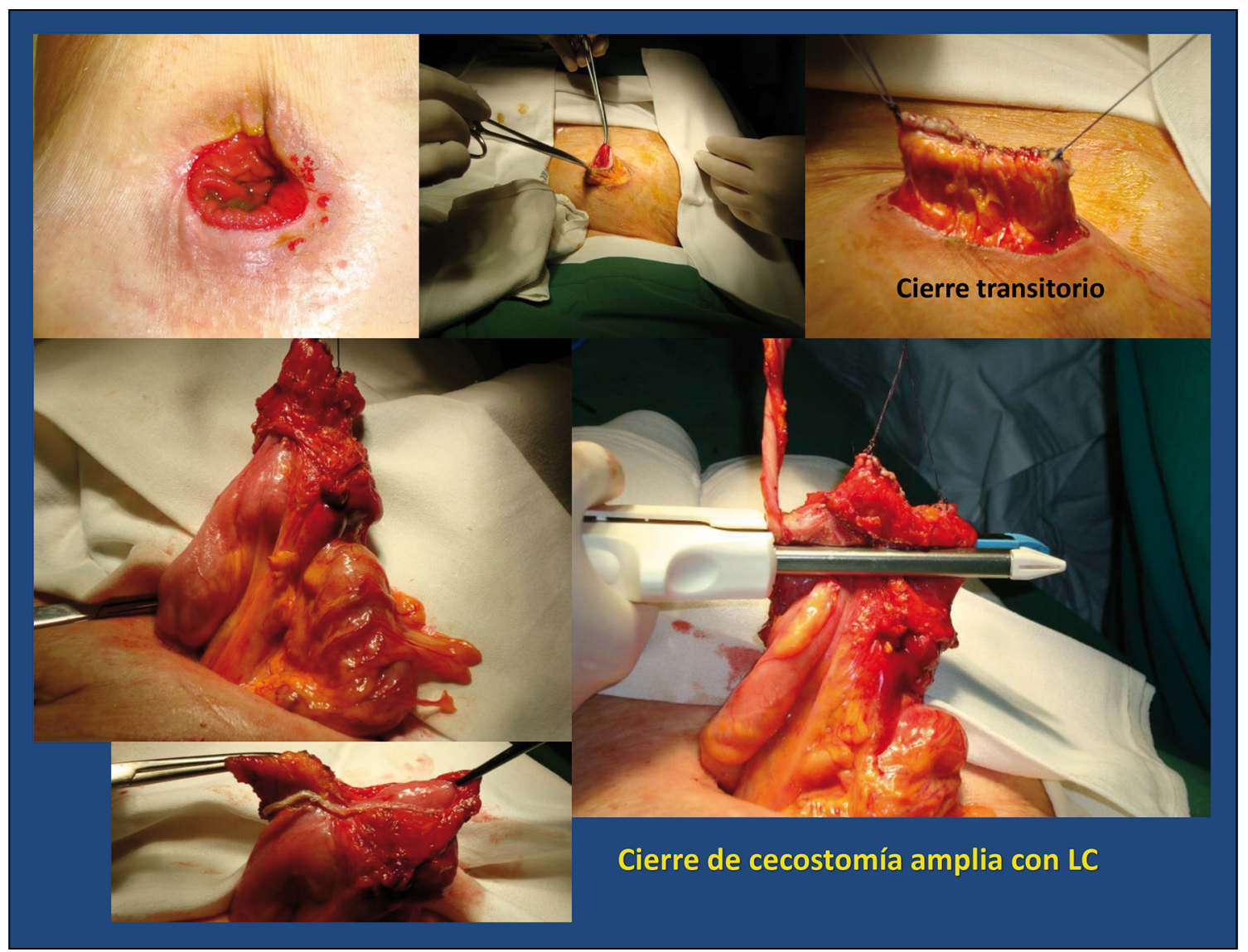

Figura 4. Cierre de la cecostomía (sutura mecánica).

Tabla 2. Resultados quirúrgicos y etapificación definitiva según tipo de ostomía de protección

\begin{tabular}{|c|c|c|}
\hline Variable & $\begin{array}{c}\text { Cecostomía } \\
n=12\end{array}$ & $\begin{array}{c}\text { Ileostomía } \\
\mathrm{n}=12\end{array}$ \\
\hline Altura anastomosis promedio $\mathrm{cm}$ & 5,4 & 6 \\
\hline RAB/RAUB* & $4 / 8$ & $8 / 4$ \\
\hline $\begin{array}{l}\text { Morbilidad mayor específica: } \\
\text { Íleo prolongado } \\
\text { Fístula }\end{array}$ & $\begin{array}{l}0 \\
3\end{array}$ & $\begin{array}{l}5 \\
3\end{array}$ \\
\hline $\begin{array}{l}\text { Días de hospitalización promedio } \\
\text { (extremos) }\end{array}$ & $\begin{array}{c}10,4 \\
(4-22)\end{array}$ & $\begin{array}{c}18 \\
(5-62)\end{array}$ \\
\hline Reoperaciones & 0 & 0 \\
\hline $\begin{array}{l}\text { Estadio patológico } \\
\text { I/IIA/IIB/IIIB/IV }\end{array}$ & $4 / 4 / 0 / 3 / 1$ & $4 / 5 / 1 / 2 / 0$ \\
\hline
\end{tabular}

RAB: Resección anterior baja. RAUB: Resección anterior ultrabaja. fue de 50 min (extremos 35-60) para el grupo CA y 49 min (extremos 30-90) para el grupo IA. El tiempo de hospitalización promedio fue 3,5 días (extremos 2-5) para el grupo CA y 5,3 días (extremos 4-8) para el grupo IA $(\mathrm{p}<0,05)$ y no hubo reoperaciones. No hubo fístulas ni infección del sitio operatorio en el grupo CA y sólo se registra un seroma de la herida operatoria. En el grupo IA hubo 1 caso de íleo postoperatorio moderado que prolonga la hospitalización y 1 caso de infección del sitio operatorio.

\section{Discusión}

Existen escasos estudios que analizan el rol de la cecostomía de tubo en la protección de una anastomosis colorrectal, con resultados muy disímiles $^{8}$, técnica que difiere de la CA motivo de este estudio. Luego de haber observado la buena 
evolución de algunos pacientes sometidos a una CA de necesidad en el contexto de una urgencia obstructiva, iniciamos este estudio prospectivo no randomizado para estudiar la eficacia de la técnica de la CA como protección de una anastomosis baja. Contrariamente a lo enseñado por décadas, la CA, por definición una colostomía lateral, ha logrado en nuestra experiencia una adecuada desfuncionalización del tracto colónico distal, ofreciendo una protección satisfactoria de una anastomosis colorrectal baja, al menos comparable con la IA.

Las ventajas durante la confección de la CA son difíciles de demostrar debido a la complejidad y la morbilidad propia de la cirugía radical, que puede ser difícil de separar de las complicaciones específicas de la ostomía. Sin embargo, al comparar ambos grupos se aprecia una reducción significativa de la estadía hospitalaria en los pacientes sometidos a una CA en comparación con la IA. La causa de la disfunción de una ileostomía debidamente evertida y madurada es motivo de debate. En nuestra opinión, la excesiva angulación del íleon terminal exteriorizado como ileostomía en asa, movilización que está limitada por un meso relativamente corto, es uno de los factores principales de este fenómeno, que es particularmente intenso en los pacientes obesos y/o de pared gruesa.

La ejecución de una $\mathrm{CA}$ es claramente más simple y de menor tiempo quirúrgico que una IA. Esto se explica porque, si bien la contrabertura para exteriorizar el intestino es similar en ambas técnicas, la CA madura la ostomía a la piel sin otros medios de fijación. Aunque no es una técnica compleja, la IA requiere una eversión asimétrica de la pared intestinal y la fijación con puntos sero-musculares para lograr la necesaria protrusión de la ileostomía.

En el seguimiento hemos constatado un caso de prolapso moderado de una $\mathrm{CA}$, con visualización de la válvula ileocecal, aunque sin alteración de la función evacuatoria. A pesar de ser una ostomía plana y de estar tan cerca del íleon, no hemos observado problemas especiales en el manejo de la ostomía en los casos de CA, que requiere los mismos cuidados que cualquier ostomía "húmeda", con episodios de dermitis periostómico transitorios que no han impedido la adecuada adhesión de la placa protésica a la piel.

Las ventajas en el cierre de la CA eran previsibles, dada la envergadura del ciego y la ausencia de asas intestinales cercanas, lo que se refleja en una reducción moderada pero significativa de la estadía hospitalaria. El cierre con sutura mecánica aunque muy simple en su ejecución, debe reparar en la vál- vula ileocecal que no debe quedar incluida en la sutura. La liberación de la IA es compleja en algunos casos debido al intenso proceso adherencial a la pared y a las asas intestinales, por lo que no es casual que en todas las series de cierre de ileostomías se reporten casos de perforación intestinal inadvertida con peritonitis postoperatoria ${ }^{3-5}$.

En conclusión, en este estudio preliminar con un número limitado de pacientes, la CA se aprecia como una alternativa válida como método de protección de una anastomosis colorrectal en pacientes intervenidos por un cáncer de recto bajo, con algunas ventajas sobre la ileostomía por la facilidad en la ejecución y en el cierre, con una reducción del tiempo de hospitalización en ambos tiempos quirúrgicos y la ausencia de íleo prolongado comparado con la IA. Por tratarse de un estudio no randomizado, estos resultados requieren comprobarse en un trabajo aleatorio con un número mayor de casos, idealmente en un estudio multicéntrico que permita evaluar la reproductibilidad de la técnica.

\section{Referencias}

1. Bannura G, Cumsille MA, Barrera A, Contreras J, Melo C, Soto D, y cols. Factores de riesgo de dehiscencia de una anastomosis colorrectal grapada: análisis multivariado. Rev Chil Cir. 2007;59:287-92.

2. Güenaga KF, Lustosa SA, Saad SS, Saconato H, Matos D. Ileostomy or colostomy for temporary decompression of colorectal anastomosis. Cochrane database System Rev.20067;CD 004647.

3. Chow A, Tilney HS, Paraskeva P, jeyarajah S, Zacharakis E, Purkayastha $\mathrm{S}$. The morbidity surrounding reversal of defunctioning ileostomies: a systematic review of 48 studies including 6.107 cases. Int J Colorectal Dis. 2009;24:711-23.

4. Suárez J, Zúñiga C, Ahumada F, Cifuentes C, Maturana F. Complicaciones del cierre de ileostomía en asa. Rev Chil Cir. 2004;56:558-61.

5. Barrera A. Cierre de ileostomía en asa con sutura mecánica. Rev Chil Cir. 2003;55:573-9.

6. Bannura G, Barrera A, Melo C, Contreras J, Soto D. Resultados del tratamiento con neoadyuvancia del cáncer de recto localmente avanzado. Rev Chil Cir. 2010;62:607-13.

7. Dindo D, Demartines N, Clavien P-A. Classification of surgical complications. A new proposal with evaluation in a cohort of 6.336 patients. Ann Surg. 2004;240:20513.

8. Saber A, Hokkam EN. Efficacy of protective tube cecostomy after restorative resection for colorectal cancer: a randomized trial. Int J Surg. 2013;11:350-3. 